\title{
Tecnologias em saúde aplicadas na reabilitação de mulheres com câncer de mama: revisão de escopo
}

\section{Health technologies applied in the rehabilitation of women with breast cancer: scoping review}

\author{
(D) Mariele Lenhari Gonçalves ${ }^{1}$, (D)Aniele Tomadon ${ }^{1}$, [Dóris Aparecida Prado da Cruz ${ }^{1}$, (DThaís de \\ Oliveira Gozzo ${ }^{1}$
}

\section{RESUMO}

Objetivo: Identificar as tecnologias em saúde na reabilitação física de mulheres que desenvolveram alterações no membro superior homolateral após a cirurgia para o câncer de mama. Método: Trata-se de uma Scoping literature review. Realizou-se a busca nas bases de dados LILACS, PubMed e Web of Science e o mecanismo de busca Google Scholar. Os descritores utilizados foram: Breast neoplasms, Biomedical technology e Rehabilitation. Resultados: Foram selecionados 33 estudos. Entre as tecnologias leve-duras, a mais utilizada foram os programas de exercícios físicos, tanto para a limitação da amplitude de movimentos do membro superior quanto para o linfedema, sendo estas as mais estudadas. Já as tecnologias duras mais avaliadas foram acupuntura, terapia de laser de baixo nível e plataformas de exercício on line, sendo empregadas, principalmente, para complicações como dor e linfedema. Destaque para a inter-relação entre as diferentes tecnologias, e como estas compõem a assistência prestadas às mulheres com câncer de mama durante a reabilitação física. Conclusão: A maioria dos estudos utilizaram as tecnologias leveduras, com destaque para os programas de exercícios, e a associação de diferentes tecnologias, de intensidade variadas, empregadas com bons resultados físicos. Além de atuarem no estímulo ao autocuidado e na orientação das mulheres quanto a autogestão das complicações, promovendo a autonomia, confiança e retomada de seus papéis sociais.

Palavras-chave: Neoplasias da Mama, Tecnologia Biomédica, Reabilitação

${ }^{1}$ Escola de Enfermagem de Ribeirão Preto da Universidade de São Paulo - EERP-USP

\section{Correspondência}

Thaís de Oliveira Gozzo

E-mail: thaisog@eerp.usp.br

Recebido: 14 Abril 2020

Aceito: 24 Junho 2020

\section{Como citar}

Gonçalves ML, Tomadon A, Cruz LAP, Gozzo TO. Tecnologias em saúde aplicadas na reabilitação de mulheres com câncer de mama: revisão de escopo. Acta Fisiatr. 2020;27(1):51-57.

DOI: 10.11606/issn.2317-0190.v27i1a168763

\begin{abstract}
Objective: To identify health technologies in the physical rehabilitation for women who developed changes on the same side upper limb after surgery for breast cancer. Method: This is a Scoping literature review. The search was performed in the LILACS, PubMed and Web of Science databases and the Google Scholar search engine. The descriptors used were: Breast neoplasms, Biomedical technology and Rehabilitation. Results: Thirty-three studies were selected. Among the soft-hard technologies, the most frequently used were the physical exercise programs, both for limiting the range of motion of the upper limb and for lymphedema, these being the most studied. The most evaluated hard technologies were acupuncture, low level laser therapy and online exercise platforms, being used mainly for complications such as pain and lymphedema. We Highlight the interrelationship between different technologies and how they provide assistance to women with breast cancer during physical rehabilitation. Conclusion: Most studies used light-hard technologies, with emphasis on exercise programs, and the association of different technologies of varying intensities are used with good physical results. It was also observed that the avalilables technologies stimulate self-care and guide women regarding self-management of complications, promoting autonomy, confidence and resumption of their social roles.
\end{abstract}

Keywords: Breast Neoplasms, Biomedical Technology, Rehabilitation 


\section{INTRODUÇÃO}

O aumento da incidência do câncer de mama observado no mundo ocidental, combinado com o tempo de sobrevida prolongado, proporcionou a organização de políticas de detecção precoce da doença e a combinação de tratamentos e terapias na busca da cura do câncer. ${ }^{1,2}$ Os tratamentos projetados para salvar ou prolongar a vida das mulheres podem causar impactos psicológicos, sociais e físicos que alteram a qualidade de vida de quem enfrenta a doença. ${ }^{3-5}$

Apesar de diversos tratamentos disponíveis, o procedimento cirúrgico ainda compõe uma das modalidades terapêuticas mais utilizadas para o câncer de mama. Entretanto, podem ocorrer complicações pós-operatórias que afetam negativamente, a curto e a longo prazo, as atividades de vida diária, o autocuidado, o desempenho profissional e social da mulher. Entre essas complicações, é possível observar a dor, formação de seroma, linfedema, diminuição da amplitude de movimento e força muscular do braço homolateral ao procedimento cirúrgico. ${ }^{6}$

Evidências científicas indicam que a maioria das mulheres experimenta uma ou mais dessas complicações e, assim, sofrem com a carga agregada de morbidades decorrentes dos tratamentos. ${ }^{7,8}$ Desta forma, a reabilitação tem como objetivo proporcionar a máxima independência possível, melhorar as funções diminuídas ou perdidas, promover autoestima e o gerenciamento do autocuidado por meio de intervenções técnicas, ações interdisciplinares e familiares, dentro e fora de instituições hospitalares..$^{9,10}$

Segundo a Organização Mundial da Saúde (OMS), a reabilitação é um processo que visa permitir que os indivíduos alcancem um ideal físico, intelectual, psicológico e social. ${ }^{11}$ Atualmente, as diversas vertentes de atenção à saúde, focadas na reabilitação, são realizadas por equipe multiprofissional, com um conjunto de ações destinadas ao cuidado e que utilizam diferentes intensidades de tecnologias com o intuito de promover o bemestar físico e mental. ${ }^{12-13}$

O processo de reabilitação em mulheres que tiveram câncer de mama, além de lidar com os efeitos negativos dos tratamentos concluídos ou em curso, visa também auxiliálas na superação de possíveis impactos psicológico, do diagnóstico e da terapêutica, como o medo da reincidência da doença e implicações sociais. ${ }^{14}$ É fundamental que haja uma avaliação sistemática em relação ao tipo de reabilitação, intervenção e tecnologias em saúde preconizadas para as mulheres com câncer de mama, uma vez que o câncer é uma doença dinâmica, e a reavaliação frequente do objetivo da reabilitação é fundamental para alcançar o bem-estar geral. ${ }^{15}$

O Conselho Nacional de Saúde (CNS) considerou todos os aspectos do tratamento do câncer, a fim de atender às necessidades na melhoria de diagnósticos, dos tratamentos e da reabilitação. A partir desta necessidade, elaboraram uma revisão sistemática para avaliar a tecnologia médica, buscando estratégias para combater a depressão, aumentar o estímulo à atividade física ou abordar alguns dos efeitos tardios dos cânceres de mama, próstata e colón e reto. Tal análise concluiu que existe carência de evidências científicas quanto às intervenções que devem ser empregadas no decorrer do tratamento, bem como seus aspectos de duração e intensidade, e ações relacionadas com a reabilitação. ${ }^{16}$

As diferentes tecnologias para assistência em saúde, utilizadas e desenvolvidas ao longo dos anos, traz a reflexão sobre quais devem ser priorizadas para promover a integralidade do cuidado. O emprego dessas tecnologias favorece a organização de elementos que permitem orientar decisões estratégicas em um serviço de saúde ou assistência médica. Estas decisões não são somente sobre as formas de cuidados dispensados aos pacientes, mas também acerca da cobertura de procedimentos e da alocação de recursos. ${ }^{17}$

A justificativa deste estudo se dá pela relevância da identificação das tecnologias em saúde aplicadas na reabilitação física de mulheres com câncer de mama no período pós-operatório, uma vez que as complicações surgidas neste período afetam desfavoravelmente a mulher no âmbito biopsicossocial e requerem estratégias de cuidados consistentes com tais necessidades.

\section{OBJETIVO}

Identificar as tecnologias em saúde na reabilitação física de mulheres que desenvolveram alterações no membro superior homolateral após a cirurgia para o câncer de mama.

\section{MÉTODO}

Para o desenvolvimento deste estudo foi realizado uma scoping review (SR) como proposto por Arkeye O'Malley. ${ }^{18}$ A estratégia SR fundamenta-se em uma revisão sistematizada e exploratória com o intuito de investigar a produção científica sobre determinadas áreas ou temáticas. ${ }^{18}$ Esta metodologia propõe as seguintes etapas, que foram seguidas neste estudo: 1) identificação da pergunta ou das perguntas da pesquisa; 2) identificação dos estudos relevantes; 3 ) seleção dos estudos; 4) extração de dados; 5) síntese e descrição dos resultados; e 6) divulgação.

A primeira etapa iniciou com a seguinte pergunta: Quais as tecnologias em saúde estão em sendo empregadas na reabilitação física de mulheres que desenvolveram alterações no membro superior homolateral após a cirurgia para o câncer de mama?

Buscando responder esta questão, passouse para a segunda etapa, caracterizada pela identificação dos estudos. A busca foi realizada em abril de 2019 nas bases de dados pelo método on line: United States National Library of Medicine (PubMed), Literatura LatinoAmericana e do Caribe em Ciências da Saúde (LILACS) e Web of Science e o mecanismo de busca Google Scholar. Em cada base, para a realização das buscas, foram utilizados os descritores controlados e seus sinônimos: Breast neoplasms, Biomedical technology e Rehabilitation com os conectores booleanos AND e OR. Além disso, foi especificado que os campos título e/ou resumos estivessem nos idiomas português, inglês e espanhol. Em relação ao intervalo de tempo, optou-se por não limitar a busca por este fator.

Para a terceira etapa, a seleção dos artigos, utilizou-se como critérios de inclusão: artigos primários publicados na íntegra, nos quais a amostra fosse composta exclusivamente por mulheres adultas ( $\geq 18$ anos), com câncer de mama em reabilitação física em decorrência de alterações no membro superior homolateral após a cirurgia. Para garantir a possibilidade de extrapolar as conclusões para este grupo populacional especifico, foram definidos como critérios de exclusão os artigos que incluíram homens, que as alterações de membro superior não estivessem relacionadas ao câncer de mama e estudos com mulheres com câncer de mama metastático.

A busca resultou em 2.817 publicações, foram excluídos 86 duplicados. Após a seleção, 84 foram lidos na integra e a amostra final foi de 33 estudos (Figura 1). ${ }^{19}$ Todas as etapas foram feitas independentemente pelos autores e as discordâncias foram resolvidas em consenso por meio dos critérios de inclusão e exclusão.

A partir dos estudos selecionados, foi realizada a extração dos dados, referente à quarta etapa e, em seguida, cumpriu-se 


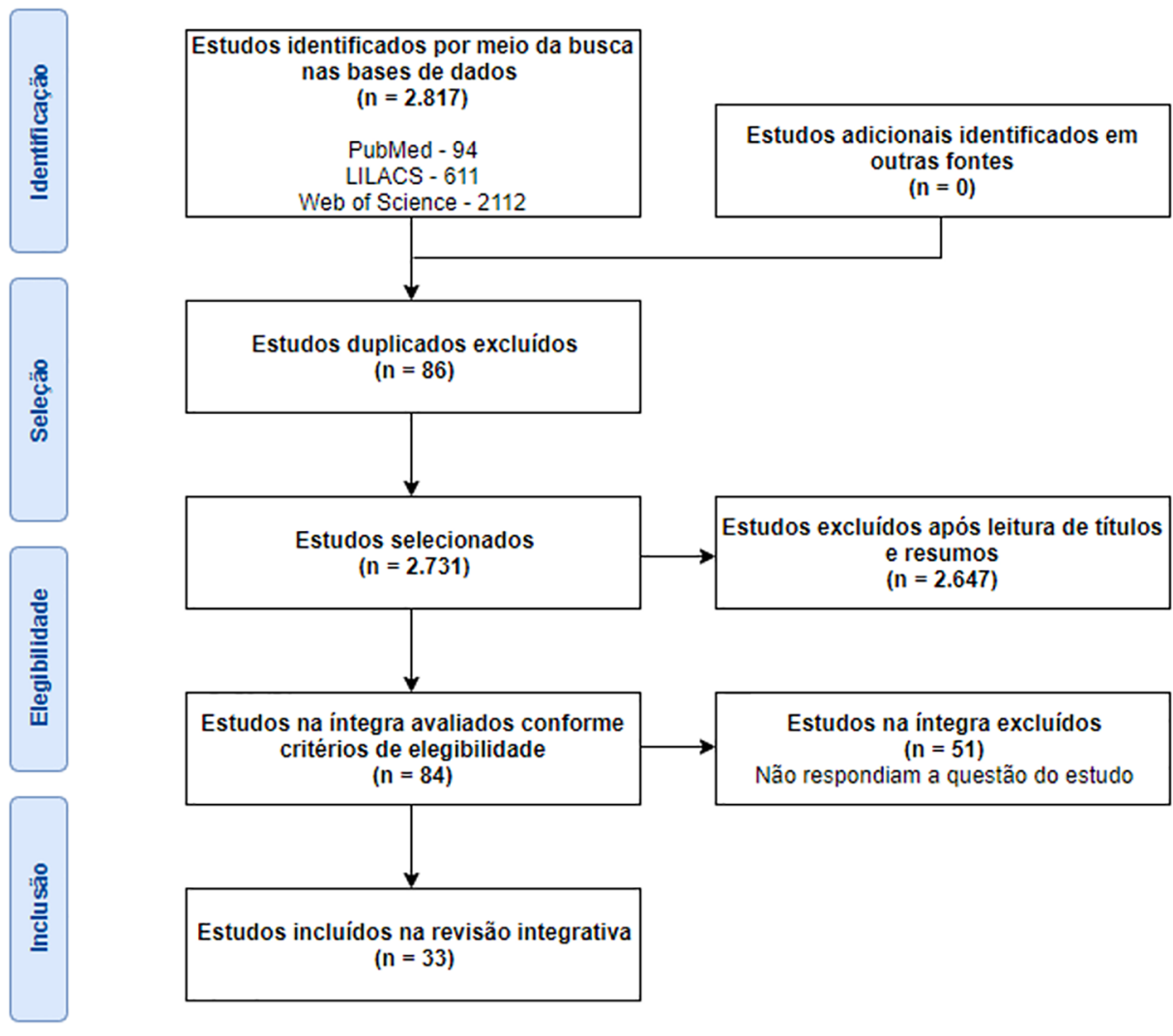

Figura 1. Fluxograma de identificação, seleção e inclusão dos estudos

a quinta etapa, de agrupamento e síntese dos resultados. Para apresentar uma visão geral do material analisado, optou-se por apresentar as tecnologias em saúde de acordo com a classificação: leves, leve-duras e duras. As tecnologias leves compreendem as relações interpessoais, como a produção de vínculos e conhecimento; as leve-duras dizem respeito aos saberes bem estruturados, como a clínica médica, e as duras são compostas por equipamentos tecnológicos do tipo máquina, normas e estruturas organizacionais. ${ }^{20}$

\section{RESULTADOS}

Os 33 artigos selecionados foram divididos de acordo com a classificação das tecnologias utilizadas. Para uma visão geral destes estudos, pode-se observar que o período de publicação ficou entre os anos de 2004 a 2018, o país com o maior número de publicações foi o Brasil (13), a categoria profissional predominante foi de fisioterapeuta (26) e 15 estudos foram desenvolvidos em hospitais universitários, (Tabela 1).

Quanto à classificação das tecnologias, observou-se em 26 estudos as tecnologias leve-duras (Quadro 1 e Quadro 2) e em sete o uso de tecnologias duras (Quadro 3). Não foram identificados estudos que empregaram tecnologias leves no manejo de complicações relacionadas ao membro superior após o câncer de mama.

Entre as tecnologias leve-duras, a complicação pós-operatória mais apontada pelos estudos incluídos foi a limitação da amplitude de movimentos do membro superior, com 15 estudos. Entre as técnicas abordadas, é possível verificar a importância dos programas de exercícios físicos expressa pela maioria dos estudos ${ }^{21,27-29,32,33,43}$ para a melhora da limitação, bem como outras estratégias como: liberação miofascial, ${ }^{42-44}$ Hatha-yoga, ${ }^{37}$ cinesioterapia, ${ }^{38}$ fisioterapia

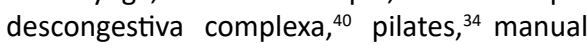
ilustrativo ${ }^{22}$ e a drenagem linfática associada à facilitação neuromuscular. ${ }^{45}$

A segunda complicação mais citada foi o linfedema, com 11 artigos. Apontou-se os exercícios físicos em seis estudos ${ }^{23,26,31,36,43}$ como estratégia de tratamento e prevenção do linfedema, assim como, a realização do mesmo com carga de peso sem dano ao sistema linfático. Outra modalidade apontada foi a técnica denominada de Kinesioterapia Taping (Kinesio taping), a qual consiste no emprego de bandagens com a finalidade de melhorar o tônus muscular e estimular a drenagem linfática. ${ }^{39}$

A fisioterapia descongestiva complexa (ou terapia física complexa) também foi outra estratégia apontada pelos estudos ${ }^{35,36,41}$ e combina técnicas como a drenagem linfática manual, enfaixamento compressivo funcional, exercícios terapêuticos e contenção elástica como método de tratamento intensivo para o linfedema. $\mathrm{O}$ uso da terapia física combinada (exercícios físicos e braçadeira elástica) foi apontado em um estudo ${ }^{30}$ e o emprego da intervenção da drenagem linfática manual associada à facilitação neuromuscular foi apontado por outro estudo. ${ }^{45}$

O manejo da dor também foi citado em seis estudos que empregaram o uso de tecnologias leve-duras. Os recursos empregados foram os exercícios físicos baseados na técnica do pilates $^{(34)}$, cinesioterapia isolada, ${ }^{38}$ cinesioterapia associada à terapia manual, ${ }^{46}$ drenagem linfática manual juntamente com a facilitação neuromuscular ${ }^{45}$ e a liberação miofascial. ${ }^{42-44}$

As tecnologias leve-duras também foram utilizadas em estudos referentes à alteração da força muscular no pós-operatório com o uso programas de exercícios físicos com orientações verbais e manuais de orientação ${ }^{28}$ e exercícios baseados nas técnicas de pilates. ${ }^{34}$ A disfunção escapular foi citada em um estudo e o exercício orientado para a escápula foi a intervenção proposta. ${ }^{25}$

Em relação às tecnologias duras, sete estudos as abordaram, sendo que cinco empregaram tratamentos para o linfedema com o uso da terapia de laser de baixo nível, ${ }^{47-49}$ estimulação elétrica de alta voltagem, ${ }^{50}$ e da terapia extracorpórea por ondas de choque. ${ }^{51}$

A dor foi a segunda complicação apontada pelos estudos que podem ser manejada com apoio de tecnologias duras, como a acupuntura ${ }^{53}$ e um programa de exercícios desenvolvido por meio de um sistema on-line. ${ }^{52} \mathrm{~A}$ diminuição da amplitude de movimentos também foi descrita e a acupuntura $^{53}$ foi uma das estratégias de manejo, assim como programa de exercícios desenvolvido por meio de um sistema online ${ }^{52}$ para melhorar também a perda de força muscular após a cirurgia.

\section{DISCUSSÃO}

Observa-se que, no período pósoperatório, há uma predominância de estudos 
Tabela 1. Distribuição dos estudos incluídos segundo período de publicação, país de origem, categoria profissional e local de desenvolvimento dos estudos

\begin{tabular}{|c|c|c|}
\hline Características dos artigos & $\mathbf{n}$ & $\%$ \\
\hline \multicolumn{3}{|l|}{ Período de publicação } \\
\hline $2004-2008$ & 3 & $9 \%$ \\
\hline $2009-2013$ & 14 & $42,4 \%$ \\
\hline $2014-2018$ & 16 & $48,4 \%$ \\
\hline \multicolumn{3}{|l|}{ País de origem } \\
\hline Brasil & 13 & $39,3 \%$ \\
\hline Turquia & 5 & $15,1 \%$ \\
\hline Austrália & 3 & $9 \%$ \\
\hline Polônia & 2 & $6 \%$ \\
\hline Espanha & 2 & $6 \%$ \\
\hline Índia & 2 & $6 \%$ \\
\hline Coréia & 2 & $6 \%$ \\
\hline Estados Unidos da América & 1 & $3 \%$ \\
\hline Norvega & 1 & $3 \%$ \\
\hline China & 1 & $3 \%$ \\
\hline Não especificado & 1 & $3 \%$ \\
\hline \multicolumn{3}{|l|}{ Categoria profissional } \\
\hline Fisioterapeutas & 26 & $78,7 \%$ \\
\hline Médicos & 3 & $9 \%$ \\
\hline Fisiologistas & 2 & $6 \%$ \\
\hline Enfermeiros & 1 & $3 \%$ \\
\hline Não encontrado & 1 & $3 \%$ \\
\hline \multicolumn{3}{|l|}{ Local de desenvolvimento dos estudos } \\
\hline $\begin{array}{l}\text { Hospitais universitários/serviços prestados por instituição } \\
\text { de ensino }\end{array}$ & 15 & $45,4 \%$ \\
\hline Centros Universitários & 7 & $21,2 \%$ \\
\hline Centros de Reabilitação & 6 & $18,1 \%$ \\
\hline Centros Oncológicos & 3 & $9 \%$ \\
\hline Não especificado & 2 & $6 \%$ \\
\hline
\end{tabular}

que utilizaram tecnologias leve-duras no processo de reabilitação de mulheres com câncer de mama, sendo a maioria dessas tecnologias caracterizadas pelos programas de exercícios físicos. Os exercícios demonstraram efetividade na melhora da amplitude de movimentos, dor, linfedema e perda de força muscular, além de melhorar a qualidade de vida da mulher. Corroborando com a presente revisão, uma meta-análise de ensaios clínicos avaliou as repercussões da atividade física em mulheres sobreviventes do câncer de mama e identificou que os exercícios contribuem para a melhora das dores, fadiga e postura corporal, assim como atua na prevenção e melhora da depressão, gerando efeitos psicológicos positivos. ${ }^{54}$ Outro estudo de revisão sobre os métodos de reabilitação utilizados em mulheres com câncer de mama apontou que o exercício foi o método mais eficaz na melhora de amplitude dos membros superiores, força muscular e cicatrização da ferida operatória, entretanto, inconclusivo no alívio da fadiga e linfedema. ${ }^{15}$

Importante ressaltar que o exercício físico, pode ser associado a outras estratégias durante o processo de reabilitação, como as orientações pós-operatórias e ao emprego de equipamentos com o objetivo de aliviar os desconfortos e limitações decorrentes do procedimento cirúrgico. Salienta-se que as tecnologias leve, dura e leve-dura, relacionam-se entre si e fazem parte da assistência prestada na reabilitação, as quais exigem avaliações frequentes para suprir as necessidades apresentadas pelas mulheres. ${ }^{55}$

Outro aspecto observado foi referente às diversas estratégias para controle do linfedema, uma das principais complicações da cirurgia para o câncer de mama. Estudos apontam que a prevalência de lindefema em mulheres com câncer de mama pode variar de $6 \%$ a $49 \%,{ }^{56}$ sendo que estudo recente com 125 mulheres identificou uma prevalência de $34,4 \% .{ }^{57}$ Por representar uma temida complicação pós-operatória, gerando grande morbidade e afetando negativamente a vida da mulher, muitas estratégias de prevenção, tratamento e controle progrediram ao longo dos anos e a presente revisão mostra este resultado, na qual se observa estudos que empregaram as tecnologias leve-duras e duras como terapêutica para o linfedema.

Apesar da importância do uso das tecnologias, em associação, é preciso estabelecer, por parte da equipe multiprofissional, uma atitude reflexiva acerca do emprego adequado dessas tecnologias no cuidado às mulheres com câncer de mama com o objetivo de prestar assistência integral e efetiva. ${ }^{58}$ Assim, percebe-se a importância do emprego de tecnologias em saúde no processo de reabilitação de mulheres com câncer de mama no período pós-operatório, no qual as tecnologias devem fortalecer e aprimorar o cuidado em saúde. ${ }^{59}$ Percebe-se a necessidade de mais estudos que analisem os efeitos das tecnologias em saúde no processo de reabilitação de mulheres com câncer de mama, objetivando a ampliação do conhecimento da equipe multiprofissional quanto a importância da associação das técnicas para garantir uma melhor assistência à mulher.

\section{CONCLUSÃO}

Os resultados da pesquisa evidenciaram a abrangência de estudos que empregaram as tecnologias em saúde na reabilitação do membro superior após o câncer de mama e que contribuem para a prevenção e manejo de complicações que geram grande morbidade.

Deve-se ressaltar que, a maioria dos estudos utilizaram as tecnologias leve-duras, com destaque para programas de exercícios, apresentando resultados positivos nas diferentes complicações no membro superior homolateral pós-cirurgia do câncer de mama.

Também se observou a associação de diferentes tecnologias, de intensidade variadas empregadas com bons resultados físicos, no estimulo ao autocuidado e na orientação das mulheres quanto a autogestão das complicações, promovendo a autonomia, confiança e retomada de seus papéis sociais.

Esta revisão pode ser utilizada tanto na pesquisa quanto no cuidado, uma vez que aponta as tecnologias disponíveis e que podem ser empregadas por diferentes profissionais da saúde capacitados para sua implementação. 
Quadro 1. Caracterização dos estudos classificados como tecnologias leve-duras que utilizaram os exercícios físicos como tecnologia de saúde

\begin{tabular}{|c|c|c|}
\hline \multicolumn{3}{|r|}{ Tecnologia Leve-dura } \\
\hline Autores & Tecnologia utilizada & Resultados \\
\hline $\begin{array}{l}\text { Silva et al. }{ }^{21} \\
2004\end{array}$ & Protocolo de exercícios & $\begin{array}{c}\text { A realização dos exercícios ativos e de alongamento, com amplitude livre desde o primeiro dia de pós- } \\
\text { operatório, permitiu boa recuperação da capacidade funcional do ombro }\end{array}$ \\
\hline $\begin{array}{l}\text { Amaral et al. }{ }^{22} \\
2005\end{array}$ & Manual ilustrado de exercícios & $\begin{array}{l}\text { O manual de exercícios físicos mostrou-se efetivo, podendo ser sugerido como uma ferramenta para } \\
\text { minimizar as intercorrências pós-operatórias }\end{array}$ \\
\hline $\begin{array}{l}\text { Hayes et al. }{ }^{23} \\
2009\end{array}$ & $\begin{array}{l}\text { Programa de exercícios aeróbicos e de } \\
\text { resistência }\end{array}$ & $\begin{array}{l}\text { Não foram observadas alterações do linfedema. Mulheres com linfedema secundário devem ser } \\
\text { encorajadas a serem fisicamente ativas, otimizando sua recuperação física e psicossocial }\end{array}$ \\
\hline $\begin{array}{l}\text { Sagen et al. }{ }^{24} \\
2009\end{array}$ & Programas de exercícios & $\begin{array}{c}\text { Mulheres submetidas a linfadenectomia axilar devem ser encorajadas a manter as atividades de vida } \\
\text { diária, sem restrições ao nível de atividade física do membro afetado }\end{array}$ \\
\hline $\begin{array}{l}\text { Lee et al. }{ }^{25} \\
2010\end{array}$ & $\begin{array}{l}\text { Programa de exercícios de ombro orientado } \\
\text { para a escápula }\end{array}$ & $\begin{array}{l}\text { O exercício orientado para a escápula pode ser eficaz para melhorar a dor, a qualidade de vida e os } \\
\text { aspectos da força em sobreviventes de câncer de mama }\end{array}$ \\
\hline $\begin{array}{l}\text { Gautam et al. }{ }^{26} \\
2011\end{array}$ & $\begin{array}{l}\text { Programa domiciliar de exercícios de resistência } \\
\text { progressiva dos membros superiores }\end{array}$ & $\begin{array}{c}\text { O programa de exercícios domiciliares melhorou efetivamente o membro superior afetado e levou a uma } \\
\text { melhora na qualidade de vida das mulheres com câncer de mama }\end{array}$ \\
\hline $\begin{array}{l}\text { Petito et al. }{ }^{27} \\
2012\end{array}$ & Programa de exercícios & $\begin{array}{l}\text { O programa de exercícios foi efetivo para a recuperação dos movimentos de flexão, abdução e extensão } \\
\text { do ombro homolateral à cirurgia, bem como seu início precoce }\end{array}$ \\
\hline $\begin{array}{l}\text { Kilbreath et al. }{ }^{28} \\
2012\end{array}$ & $\begin{array}{l}\text { Programa de exercícios (manual e orientações } \\
\text { verbais) para amplitude de força muscular }\end{array}$ & $\begin{array}{l}\text { As mulheres ganharam amplitude de movimentos na flexão e abdução para a frente e força no plano de } \\
\text { abdução }\end{array}$ \\
\hline $\begin{array}{l}\text { Silva et al. }{ }^{29} \\
2013\end{array}$ & Protocolo de exercícios & $\begin{array}{c}\text { O protocolo de exercícios melhorou os movimentos do membro superior homolateral ao câncer de mama, } \\
\text { tornando-o similar ao membro contralateral }\end{array}$ \\
\hline $\begin{array}{l}\text { Ochalek et al. }{ }^{30} \\
2015\end{array}$ & $\begin{array}{l}\text { Programa de exercícios e uso de braçadeira } \\
\text { compressiva }\end{array}$ & O estudo apontou redução significativa do linfedema com as estratégias utilizadas \\
\hline $\begin{array}{l}\text { Buchan et al. }{ }^{31} \\
2016\end{array}$ & $\begin{array}{l}\text { Programa de exercícios de resistência e } \\
\text { aeróbicos para tratamento do linfedema }\end{array}$ & $\begin{array}{c}\text { Exercícios de resistência ou aeróbicos não alteraram o status do linfedema, mas levou a melhora } \\
\text { clinicamente relevante na função e na qualidade de vida }\end{array}$ \\
\hline $\begin{array}{l}\text { Petry et al. }{ }^{32} \\
2016\end{array}$ & $\begin{array}{l}\text { Protocolo de exercícios para melhorar a } \\
\text { amplitude de movimentos }\end{array}$ & $\begin{array}{l}\text { O protocolo de exercícios associado a pompage em região cervical e torácica, mobilização cicatricial e } \\
\text { sensibilização da região mamária, foram eficazes para aumento da amplitude de ombro }\end{array}$ \\
\hline $\begin{array}{l}\text { Rezende et al. }{ }^{33} \\
2017\end{array}$ & $\begin{array}{l}\text { Programa de exercícios para melhorar a } \\
\text { amplitude de movimento e postura corporal }\end{array}$ & $\begin{array}{l}\text { A aplicação de exercícios supervisionados se mostrou importante na correção de desvios posturais e na } \\
\text { recuperação da abdução de ombro }\end{array}$ \\
\hline $\begin{array}{l}\text { Alpozgen et al. }{ }^{34} \\
2017\end{array}$ & Exercícios de Pilates & $\begin{array}{l}\text { Os exercícios baseados no Pilates parecem ser efetivos na reabilitação de mulheres com transtornos na parte } \\
\text { superior do corpo (dor, perda de força muscular e diminuição da amplitude de movimentos) relacionados } \\
\text { ao tratamento do câncer de mama, e pode ser considerado como uma alternativa ao uso de cinesioterapia }\end{array}$ \\
\hline
\end{tabular}

Quadro 2. Caracterização dos estudos classificados como tecnologias leve-duras que utilizaram tecnologias diversas.

\begin{tabular}{|c|c|c|}
\hline \multicolumn{3}{|r|}{ Tecnologia Leve-dura } \\
\hline Autores & Tecnologia utilizada & Resultados \\
\hline $\begin{array}{l}\text { Karadibak et al. }{ }^{35} \\
2008\end{array}$ & Fisioterapia descongestiva complexa & A intervenção promoveu a redução do linfedema e melhorou a mobilidade do membro \\
\hline $\begin{array}{l}\text { Kim et al. }{ }^{36} \\
2010\end{array}$ & Fisioterapia complexa descongestiva & Observou-se redução do volume do braço e melhora da qualidade de vida das mulheres \\
\hline $\begin{array}{l}\text { Bernardi et al. }{ }^{37} \\
2011\end{array}$ & Hatha-Yoga & $\begin{array}{c}\text { Não houve comprovação de que a intervenção Hatha-Yoga melhora na amplitude de movimento do } \\
\text { ombro de mulheres mastectomizadas }\end{array}$ \\
\hline $\begin{array}{l}\text { Rett et al. } \\
2012\end{array}$ & Cinesioterapia & Foram contatados efeitos positivos na amplitude de movimento do membro superior e redução da dor \\
\hline $\begin{array}{l}\text { Pop et al. }{ }^{39} \\
2014\end{array}$ & Kinesiologia Taping & $\begin{array}{c}\text { A intervenção exerceu influência positiva no tratamento do linfedema, com melhora na mobilidade, na } \\
\text { força de preensão e na redução do linfedema }\end{array}$ \\
\hline $\begin{array}{l}\text { Atalay et al. }{ }^{40} \\
2015\end{array}$ & Fisioterapia descongestiva complexa & $\begin{array}{c}\text { Após a intervenção a amplitude de movimento do ombro aumentou e linfedema do braço afetado diminuiu } \\
\text { significativamente }\end{array}$ \\
\hline $\begin{array}{l}\text { Buragadda et al. }{ }^{41} \\
2015\end{array}$ & Fisioterapia descongestiva complexa & Melhora do volume, da dor e da função do membro superior homolateral a cirurgia \\
\hline $\begin{array}{l}\text { Castro-Martin et al. }{ }^{42} \\
2017\end{array}$ & Indução miofascial para controle da dor & $\begin{array}{c}\text { Uma única manobra de indução miofascial produziu diminuição da intensidade da dor e melhora da } \\
\text { amplitude de movimento do pescoço e ombro }\end{array}$ \\
\hline $\begin{array}{l}\text { Oliveira et al. }{ }^{43} \\
2017\end{array}$ & $\begin{array}{l}\text { Comparação de técnicas: } \\
\text { - Estratégias educativas associadas à } \\
\text { drenagem linfática manual } \\
\text { - Exercícios ativos supervisionados }\end{array}$ & Os efeitos na reabilitação da circunferência do braço e amplitude de movimentos são semelhantes \\
\hline $\begin{array}{l}\text { Prado Junior et al. }{ }^{44} \\
2017\end{array}$ & Técnica miofascial profunda & $\begin{array}{l}\text { A técnica miofascial profunda realizada na região de peitoral promoveu aumento da amplitude de } \\
\text { movimento e redução da dor em mulheres mastectomizadas }\end{array}$ \\
\hline $\begin{array}{l}\text { Ha et al. }{ }^{45} \\
2017\end{array}$ & $\begin{array}{l}\text { Drenagem linfática manual e facilitação } \\
\text { neuromuscular }\end{array}$ & $\begin{array}{c}\text { A combinação de drenagem linfática manual e facilitação neuromuscular induz efeitos sinérgicos potentes } \\
\text { no volume do linfedema, amplitude de movimento do ombro e dor }\end{array}$ \\
\hline $\begin{array}{l}\text { Rangon et al. }{ }^{46} \\
2018\end{array}$ & Cinesioterapia e compressão isquêmica & $\begin{array}{c}\text { A compressão isquêmica associada à cinesioterapia aumenta o limiar de dor à pressão no ponto-gatilho } \\
\text { miofascial no músculo trapézio superior e reduz a intensidade da dor em sobreviventes de câncer de mama } \\
\text { com dor miofascial }\end{array}$ \\
\hline
\end{tabular}


Quadro 3. Caracterização dos estudos classificados como tecnologias duras utilizadas

\begin{tabular}{|c|c|c|}
\hline \multicolumn{3}{|c|}{ Tecnologia Leve-dura } \\
\hline Autor & Tecnologia utilizada & Resultados \\
\hline $\begin{array}{l}\text { Lau et al. }{ }^{47} \\
2009\end{array}$ & $\begin{array}{l}\text { Terapia de laser de baixo nível para controle de } \\
\text { linfedema. }\end{array}$ & $\begin{array}{l}\text { Os achados sugeriram que a de terapia a laser de baixo nível na região axilar é } \\
\text { efetiva na redução do volume do tecido afetado e no enrijecimento tecidual }\end{array}$ \\
\hline $\begin{array}{l}\text { Kozanoglu et al. } 48 \\
2009\end{array}$ & $\begin{array}{l}\text { Compressão pneumática e terapia com laser de baixa } \\
\text { intensidade para linfedema }\end{array}$ & $\begin{array}{c}\text { Os pacientes dos dois grupos melhoraram após as intervenções. O laser de baixa } \\
\text { intensidade pode ser uma modalidade útil no tratamento do linfedema pós- } \\
\text { mastectomia }\end{array}$ \\
\hline $\begin{array}{l}\text { Ridner et al. }{ }^{49} \\
2013\end{array}$ & Terapia de laser de baixa densidade para linfedema & $\begin{array}{l}\text { Terapia de laser de baixa densidade combinada ao enfaixamento compressivo } \\
\text { pode oferecer uma opção terapêutica que economiza tempo }\end{array}$ \\
\hline $\begin{array}{l}\text { Barros et al. }{ }^{50} \\
2013\end{array}$ & $\begin{array}{l}\text { Estimulação elétrica de alta voltagem associada a } \\
\text { exercícios físicos para o linfedema }\end{array}$ & $\begin{array}{l}\text { O protocolo aplicado foi efetivo para a redução do linfedema na população } \\
\text { estudada }\end{array}$ \\
\hline $\begin{array}{l}\text { Cebicci et al. } .^{51} \\
2016\end{array}$ & $\begin{array}{l}\text { Terapia extracorpórea por ondas de choque para } \\
\text { linfedema }\end{array}$ & $\begin{array}{l}\text { Redução do linfedema e melhora acentuada do comprometimento funcional e } \\
\text { qualidade de vida }\end{array}$ \\
\hline $\begin{array}{l}\text { Galiano-Castillo et al.52 } \\
2016\end{array}$ & $\begin{array}{l}\text { Sistema on-line de exercícios para melhorar a dor e força } \\
\text { muscular }\end{array}$ & $\begin{array}{c}\text { O sistema pode melhorar a dor e a força muscular por meio da implementação } \\
\text { de um programa de exercícios sob medida, baseado na Internet. As melhorias na } \\
\text { força muscular levaram a melhorias na funcionalidade relacionadas às atividades } \\
\text { diárias }\end{array}$ \\
\hline $\begin{array}{l}\text { Giron et al. }{ }^{53} \\
2016\end{array}$ & $\begin{array}{l}\text { Acupuntura associada a cinesioterapia na reabilitação } \\
\text { de distúrbios físicos e funcionais (dor, amplitude de } \\
\text { movimento, função do membro superior) }\end{array}$ & $\begin{array}{l}\text { Melhora estatisticamente significativa dos itens avaliados: dor, função do membro } \\
\text { superior e amplitude de movimento }\end{array}$ \\
\hline
\end{tabular}

\section{REFERÊNCIAS}

1. Bray F, Ferlay J, Soerjomataram I, Siegel RL, Torre LA, Jemal A. Global cancer statistics 2018: GLOBOCAN estimates of incidence and mortality worldwide for 36 cancers in 185 countries. CA Cancer J Clin. 2018;68(6):394-424. Doi: https://doi.org/10.3322/ caac. 21492

2. Sledge GW, Mamounas EP, Hortobagyi GN, Burstein HJ, Goodwin PJ, Wolff AC. Past, present, and future challenges in breast cancer treatment. J Clin Oncol. 2014;32(19):1979-86. Doi: https://doi.org/10.1200/ JCO.2014.55.4139

3. Bushatsky M, Silva RA, Lima MTC, Barros MBSC, Beltrão Neto JEV, Ramos YTM. Qualidade de vida em mulheres com câncer de mama em tratamento quimioterápico. Cienc Cuid Saude. 2017;16(3). Doi: https://doi.org/10.4025/cienccuidsaude. v16i3.36094

4. Dalton SO, Johansen C. New paradigms in planning cancer rehabilitation and survivorship. Acta Oncol. 2013;52(2):191-194. Doi: https://doi.org/10.3109/0 284186X.2012.748216

5. Gozzo TO, Souza SG, Moysés AMB, Panobianco MS, Almeida AM. Incidence and management of chemotherapy-induced nausea and vomiting in women with breast cancer. Rev Gaucha Enferm. 2014;35(3):117-23. Doi: https://doi. org/10.1590/1983-1447.2014.03.42068

6. Fangel LMV, Panobianco MS, Kebbe LM, Almeida AM, Gozzo TO. Quality of life and daily activities performance after breast cancer treatment. Acta Paul Enferm. 2013;26(1):93-100. Doi: https://doi. org/10.1590/S0103-21002013000100015

7. Panobianco MS, Campacci N, Fangel LMV, Prado MAS, Almeida AM, Gozzo TO. Quality of life of women with lymphedema after surgery for breast cancer. Rev Rene. 2014;15(2):206-13. Doi: https:// doi.org/10.15253/2175-6783.2014000200004

8. Verbelen H, Gebruers N, Eeckhout FM, Verlinden K, Tjalma W. Shoulder and arm morbidity in sentinel node-negative breast cancer patients: a systematic review. Breast Cancer Res Treat. 2014;144(1):21-31. Doi: https://doi.org/10.1007/s10549-014-2846-5
9. Correia T, Monteiro S, Torres A, Pereira A, Caetano T, Silva R. Effectiveness of a multidisciplinary intervention in breast cancer survivor. Psic Saúde \& Doenças. 2016;17(3):483502. Doi: http://dx.doi.org/10.15309/16psd170314

10. Farias LMA, Aguiar VCF, Carvalho AMF, Linhares JM, Linhares AEMS, Sousa AMM. Group of mastectomized women: building care strategies. SANARE. 2015;14(2):91-7.

11. World Health Organization. Relatório mundial sobre a deficiência. São Paulo: SEDPcD; 2012.

12. Shoeller SD, Padilha MICS, Ramos FRS, Silva DMGV, Leopardi MT, Lorenzini A. Pesquisa em enfermagem de reabilitação: apontamentos da realidade brasileira. In: Gomes B, Rocha MC, Martins MM, Gonçalves MN. Investigação em enfermagem de reabilitação: um novo conhecimento para guiar a prática de cuidados. Porto: Escola Supeior de Enfermagem do Porto; 2014. p. 36-45.

13. Varnfield $M$, Karunanithi $M$, Lee $C K$, Honeyman $E$, Arnold D, et al. Smartphone-based home care model improved use of cardiac rehabilitation in postmyocardial infarction patients: results from a randomised controlled trial. Heart. 2014;100(22):1770-1779. Doi: https://doi. org/10.1136/heartjnl-2014-305783

14. Loyola EAC, Borges ML, Magalhães PAP, Areco FS, Yochimochi LTB, Panobianco MS. Rehabilitation group: benefits and barriers in the perspective of women with breast cancer. Texto Contexto Enferm. 2017;26(1):e3250015. Doi: https://doi. org/10.1590/0104-07072017003250015

15. Loh SY, Musa AN. Methods to improve rehabilitation of patients following breast cancer surgery: a review of systematic reviews. Breast Cancer. 2015;11(7):8198. Doi: https://doi.org/10.2147/BCTT.S47012

16. Hellbom $M$, Bergelt $C$, Bergenmar M, Gijsen B, Loge $\mathrm{JH}$, Rautalahti $\mathrm{M}$, et al. Cancer rehabilitation: a Nordic and European perspective. Acta Oncol. 2011;50(2):179-86. Doi: https://doi.org/10.3109/02 84186X.2010.533194

17. Kalichman AO, Ayres JRCM. Comprehensiveness and healthcare technologies: a narrative on conceptual contributions to the construction of the comprehensiveness principle in the Brazilian Unified National Health System. Cad Saúde Pública. 2016;32(8):1-13. Doi: https://doi.org/10.1590/0102$311 \times 00183415$
18. Arksey H, O'Malley L. Scoping studies: towards a methodological framework. Int J Soc Res Methodol. 20038(1):19-32. Doi: https://doi. org/10.1080/1364557032000119616

19. Moher D, Liberati A, Tetzlaff J, Altman DG, The PRISMA Group. Preferred reporting items for systematic reviews and meta-analyses: the PRISMA statement. PLoS Med. 2009;6(6):e1000097. Doi: https://doi. org/10.7326/0003-4819-151-4-200908180-00135

20. Merhy EE. Em busca de ferramentas analisadoras das tecnologias em saúde: a informação e o dia a dia de um serviço, interrogando e gerindo trabalho em saúde. In: Merhy EE, Onocko R. Agir em saúde: um desafio para o público. São Paulo: Hucitec; 1997. p. 113-60.

21. Silva MPP, Derchain SFM, Rezende L, Cabello C, Martinez EZ. Shoulder movement after surgery for invasive breast carcinoma: randomized controlled study of postoperative exercises. Rev Bras Ginecol Obstet. 2004;26(2):125-30. Doi: https://doi. org/10.1590/S0100-72032004000200007

22. Amaral MTP, Teixeira LC, Derchain SFM, Nogueira MD, Silva MPP, Gonçalves AV. Orientação domiciliar: proposta de reabilitação física para mulheres submetidas à cirurgia por câncer de mama. Rev Ciênc Méd. 2005;14(5):405-13.

23. Hayes SC, Reul-Hirche H, Turner J. Exercise and secondary lymphedema: safety, potential benefits, and research issues. Med Sci Sports Exerc. 2009;41(3):483-9. Doi: https://doi.org/10.1249/MSS.0b013e31818b98fb

24. Sagen A, Resen RK, Risberg MA. Physical activity for the affected limb and arm lymphedema after breast cancer surgery. A prospective, randomized controlled trial with two years follow-up. Acta Oncol. 2009;48(8):1102-10. Doi: https://doi.org/10.3109/02841860903061683

25. Lee SA, Kang JY, Kim YD, An AR, Kim SW, Kim YS, et al. Effects of a scapula-oriented shoulder exercise programme on upper limb dysfunction in breast cancer survivors: a randomized controlled pilot trial. Clin Rehabil. 2010;24(7):600-13. Doi: https://doi. org/10.1177/0269215510362324

26. Gautam AP, Maiya AG, Vidyasagar MS. Effect of home-based exercise program on lymphedema and quality of life in female postmastectomy patients: pre-post intervention study. J Rehabil Res Dev. 2011;48(10):1261-8. Doi: https://doi.org/10.1682/ JRRD.2010.05.0089 
27. Petito EL, Nazário ACP, Martinelli SE, Facina G, Gutiérrez MGR. Application of a domicile-based exercise program for shoulder rehabilitation after breast cancer surgery. Rev Latino-Am Enferm. 2012;20(1):1-9. Doi: https://doi.org/10.1590/S010411692012000100006

28. Kilbreath SL, Refshauge KM, Beith JM, Ward LC, Lee M, Simpson JM, et al. Upper limb progressive resistance training and stretching exercises following surgery for early breast cancer: a randomized controlled trial. Breast Cancer Res Treat. 2012;133(2):667-76. Doi: https://doi.org/10.1007/s10549-012-1964-1

29. Silva MD, Rett MT, Mendonça $A C R$, Silva Júnior WM, Prado VM, Santana JM. Quality of life and shoulder motion after surgery for breast cancer: physical therapy fobarcus. Rev Bras Cancerol. 2013;59(3):419-26.

30. Ochalek K, Gradalski T, Szygula Z. Five-year assessment of maintenance combined physical therapy in postmastectomy. Lymphat Res Biol. 2015;13(1):1-13. Doi: https://doi.org/10.1089/ Irb.2014.0027

31. Buchan J, Janda M, Box R, Schmitz K, Hayes S. A randomized trial on the effect of exercise mode on breast cancer-related lymphedema. Med Sci Sports Exerc. 2016;48(10):1866-74. Doi: https://doi. org/10.1249/MSS.0000000000000988

32. Petry DM, Honório JGS, Santos K, Santos S, Luz CM, Luz SCT, et al. Intervention effects on shoulder physiotherapeutic range of motion and old map thermography submitted to surgery for breast cancer treatment. Acta Fisiatr. 201623(4):180-185. Doi: https://doi.org/10.5935/0104-7795.20160034

33. Rezende MS, Gradim CVC, Berdu NR, lunes DH. Análise postural fotogramétrica após exercícios supervisionados em mulheres pós-cirurgia oncológica mamária. ConScientiae Saúde. 2017;16(1):42-9. Doi: https://doi.org/10.5585/ConsSaude.v16n1.6847

34. Alpozgen AZ, Ozdincler AR, Karanlik H, Agaoglu FY, Narin AN. Effectiveness of Pilates-based exercises on upper extremity disorders related with breast cancer treatment. Eur J Cancer Care. 2017;26(6):1-8. Doi: https://doi.org/10.1111/ecc.12532

35. Karadibak Karadibak D, Yavuzsen T, Saydam S. Prospective trial of intensive decongestive physiotherapy for upper extremity lymphedema. J Surg Oncol. 2008;97(7):572-7. Doi: https://doi. org/10.1002/jso.21035

36. Kim DS, Sim YJ, Jeong HJ, Kim GC. Effect of active resistive exercise on breast cancer-related lymphedema: a randomized controlled trial. Arch Phys Med Rehabil. 2010;91(12):1844-8. Doi: https:// doi.org/10.1016/j.apmr.2010.09.008

37. Bernardi MLD, Amorim MHC, Zandonade E, Santaella DF, Barbosa JA. Análise da amplitude de movimento dos ombros antes e após a intervenção Hatha-Yoga em mulheres mastectomizadas. Rev Bras Mastologia. 2011;21(3):118-26. Doi: https://doi.org/10.5327/ z0104-80582011000300005
38. Rett MT, Mesquita PJ, Mendonça ARC, Moura DP, Santana JM. Kinesiotherapy decreases upper limb pain in females submitted to mastectomy or quadrantectomy. Rev Dor. 2012;13(3):201-17. Doi: https://doi.org/10.1590/S1806-00132012000300002

39. Pop TB, Karczmarek-Borowska B, Tymczak M, Hałas I, Banaś J. The influence of Kinesiology Taping on the reduction of limphoedema among womag after mastectomy- preliminary study. Contemp Oncol (Pozn). 2014;18(2):124-9. Doi: https://doi. org/10.5114 / wo.2014.40644

40. Atalay OT, Özkir A, Çalik BB, Baskan E, Taşkin H. Effects of phase I complex decongestive physiotherapy on physical functions and depression levels in breast cancer related lymphedema. J Phys Ther Sci. 2015;27(3):865-70. Doi: https://doi.org/10.1589/ jpts. 27.865

41. Buragadda S, Alhusaini AA, Melam GR, Arora N. Effect of complete decongestive therapy and a home program for patients with post mastectomy lymphedema. J Phys Ther Sci. 2015;27(9):2743-8. Doi: https://doi.org/10.1589/jpts.27.2743

42. Castro-Martín E, Ortiz-Comino L, Gallart-Aragón T, Esteban-Moreno B, Arroyo-Morales M, GalianoCastillo N. Myofascial induction effects on neck-shoulder pain in breast cancer survivors: randomized, single-blind, placebo-controlled crossover design. Arch Phys Med Rehabil. 2017;98(5):832-40. Doi: https://doi.org/10.1016/j. apmr.2016.11.019

43. Oliveira MMF, Gurgel MSC, Amaral MTP, Amorim BJ, Ramos CD, Almeida Filho JG, et al. Manual lymphatic drainage and active exercise effects on lymphatic function do not translate into morbidities in women who underwent breast cancer surgery. Arch Phys Med Rehabil. 2017;98(2):256-63. Doi: https://doi.org/10.1016/j. apmr.2016.06.024

44. Prado Junior JRA, Inocêncio KR, Silva AC, Almeida MS, Bergmann A, Silva JG. Efeito imediato da técnica de mobilização nas interfaces fasciais profundas da região peitoral em pacientes submetidas à mastectomia. Fisioter Bras. 2017;18(2):180-8.

45. Ha KJ, Lee SY, Lee H, Choi SJ. Synergistic effects of proprioceptive neuromuscular facilitation and manual lymphatic drainage in patients with mastectomy-related lymphedema. Front Physiol. 2017;8:959. Doi: https://doi.org/10.3389/ fphys.2017.00959

46. Rangon FB, Ferreira VTK, Rezende MS, Apolinário A, Ferro AP, Guirro ECO. Ischemic compression and kinesiotherapy on chronic myofascial pain in breast cancer survivors. J Bodyw Mov Ther. 2018;22(1):6975. Doi: https://doi.org/10.1016/j.jbmt.2017.04.005

47. Lau RW, Cheing GL. Managing postmastectomy lymphedema with low-level laser therapy. Photomed Laser Surg. 2009;27(5):763-9. Doi: https://doi. org/10.1089/pho.2008.2330
48. Kozanoglu E, Basaran S, Paydas S, Sarpel T. Efficacy of pneumatic compression and low-level laser therapy in the treatment of postmastectomy lymphoedema: a randomized controlled trial. Clin Rehabil. 2009;23(2):11724. Doi: https://doi.org/10.1177/0269215508096173

49. Ridner SH, Poage-Hooper E, Kanar C, Doersam JK, Bond SM, Dietrich MS. A pilot randomized trial evaluating low-level laser therapy as an alternative treatment to manual lymphatic drainage for breast cancer-related lymphedema. Oncol Nurs Forum. 2013;40(4):383-93. Doi: https://doi.org/10.1188/13.ONF.383-393

50. Barros VM, Panobianco MS, Almeida AM, Guirro ECO. Post-mastectomy lymphedema: a treatment protocol. Fisioter Pesq. 2013;20(2):178-83. Doi: https://doi. org/10.1590/S1809-29502013000200013

51. Cebicci MA, Sutbeyaz ST, Goksu SS, Hocaoglu S, Oguz A, Atilabey A. Extracorporeal shock wave therapy for breast cancer-related lymphedema: a pilot study. Arch Phys Med Rehabil. 2016;97(9):1520-5. Doi: https://doi.org/10.1016/j.apmr.2016.02.019

52. Galiano-Castillo N, Cantarero-Villanueva I, FernándezLao C, Ariza-García A, Díaz-Rodríguez L, Del-Moral-Ávila $\mathrm{R}$, et al. Telehealth system: A randomized controlled trial evaluating the impact of an internet-based exercise intervention on quality of life, pain, muscle strength, and fatigue in breast cancer survivors. Cancer. 2016;122(20):3166-3174. Doi: https://doi.org/10.1002/ cncr.30172

53. Giron PS, Haddad CA, Rizzi SKLA, Nazário AC, Facina G. Effectiveness of acupuncture in rehabilitation of physical and functional disorders of women undergoing breast cancer surgery. Support Care Cancer. 2016;24(6):2491-6. Doi: https://doi.org/10.1002/cncr.30172

54. Fong DYT, Ho JWC, Hui BP, Lee AM, Macfarlane DJ, Leung SK, et al. Physical activity for cancer survivors: metaanalysis of randomized controlled trials. BMJ. 2012;34470. Doi: https://doi.org/10.1007/s00520-015-3054-5

55. Rossi FR, Lima MADS. Fundaments for managing process in care practices. Rev Esc Enferm USP. 2005;39(4):460-8. Doi: https://doi.org/10.1590/ S0080-62342005000400013

56. Paiva DMF, Rodrigues VO, Cesca MG, Palma PV, Leite ICG. Prevalence of lymphedema in women undergoing treatment for breast cancer in a referral center in south eastern Brazil. BMC Womens Health 2013;13(6):13-6. Doi: https://doi.org/10.1186/14726874-13-6

57. Bonisson PLV, Fu MR, Matos SS, Simino GPR, Lima ERP, Ercole FF. Lymphedema in women undergoing breast cancer surgery. Rev Rene. 2017;18(3):329-36. Doi https://doi.org/10.15253/2175-6783.2017000300007

58. Lopes EM, Pinheiro AKB, Pinheiro PNC, Vieira NFC. Technology and nursing practice - a bibliographical research. Online Braz J Nurs. 2009;8(1).

59. Pereira CDFD, Pinto DPSR, Tourinho FSV, Santos VEP. Technologies and their impact on nursing care practice. R-BITS. 2013;2(4):29-37. Doi: https://doi. org/10.18816/r-bits.v2i4.3331 